\title{
Error Analysis of Camera Parameter Estimation based on Collinear Features
}

\author{
Onay Urfalıōlu, Thorsten Thormählen, Hellward Broszio and Patrick Mikulastik \\ Information Technology Laboratory (LFI), University of Hannover
}

\begin{abstract}
Feature points for camera parameter estimation are detected in noisy images. Therefore, the feature points and also the camera parameters can only be estimated with limited accuracy. In case of collinear feature points, it is possible to benefit from this geometrical regularity which results in an increased accuracy of the camera parameters. In this paper, a complete theoretical covariance propagation starting from the error of the feature points up to the error of the estimated camera parameters is performed. Additionally, by determining the Fisher information matrix the Cramer-Rao bounds for the covariance of the corrected feature point positions are determined. To demonstrate the impact of collinearity on the accuracy of the camera parameters, a covariance propagation is performed with varying feature point error covariances.
\end{abstract}

\section{Introduction}

In feature point based structure-from-motion (SFM) methods the accuracy of the estimated camera parameters depends on the accuracy of the features. The knowledge of the probability density function of the uncertainty of feature point positions enables Maximum-Likelihood (ML) estimation [4]. In this case, it is also possible to determine the expected error covariances of the estimated camera parameters [4]. In [1], Bartoli et al. propose the utilization of collinear features when appropriate, where in synthetic experiments lower covariances for the errors of estimated camera parameters are measured. In [6], a simplified error covariance propagation analysis for collinear features is presented for SFM, but the analysis is not based on optimal ML-estimation, so a fully ML-based theoretical analysis of covariance is missing.

In order to take advantage of the collinearity, this paper applies the strategy to determine an MLestimate of a (straight) line, represented by the measured feature points and correcting the feature point positions by projecting them onto the estimated line. The estimation of camera parameters is then based on the corrected feature points. Thereby, it is shown by theoretical derivation that the resulting feature points have smaller error covariances resulting in higher estimation accuracy of the camera parameters.

Several proposed methods exist for estimating lines and determining the error covariances of the line parameters and corresponding Cramer-Rao lower bounds analytically $[3,8,2]$. In this paper the covariance and Cramer-Rao bound determination of line parameters is reviewed. The main contribution is an analysis of corrected point positions including the determination of the error covariances and the Cramer-Rao bounds, which depend both on the uncertainty of the line as well as on the uncertainty of the selected point to be corrected.

The focus is on camera parameter estimation, so a complete theoretical analysis starting from the error of the feature points up to the error of the estimated camera parameters is achieved.

In the following section, the ML-estimation of the line is presented. In section 3 the error covariance propagation for the corrected feature points is analytically derived. Section 4 describes the calculation of the Fisher information matrix and the CramerRao bounds for the expected error covariances of corrected feature points. Section 5 describes briefly the propagation of the error covariances up to the camera parameters followed by section 6 where the impact of collinearity is shown in experiments. In the last section the paper is concluded.

\section{Maximum-Likelihood esti- mation of line parameters}

Assume a given set of feature points supposed to lie on a straight line, simply referred to as line in the following. Furthermore, it is assumed that their detection is erroneous so they actually are not located within the line exactly.

The goal is to determine the line by processing in- 
formation given by the feature points.

A 2D-line $\mathbf{l}$ can be described by the Hessian parameterization. A point $\mathbf{x}$ lies on a line if

$$
\mathbf{n}^{\top}(\mathbf{a}-\mathbf{x})=0
$$

where

$$
\mathbf{n}=(\cos (\phi), \sin (\phi))^{\top}
$$

is the normal vector and $\mathbf{a}$ is the base. With a homogeneous point

$$
\mathbf{x}=\left(x_{1}, x_{2}, 1\right)^{\top}
$$

and

$$
\mathbf{l}=(\cos (\phi), \sin (\phi),-\rho)^{\top}
$$

the homogeneous parameterization of the line is obtained, satisfying

$$
\mathbf{l}^{\top} \mathbf{x}=0 .
$$

It is assumed that the probability density function (PDF) describing the uncertainty of the feature points is (uncorrelated) Gaussian and the covariances are known. The error covariances can be determined by analyzing the feature tracking method. E.g. for the KLT tracking method [10, 7], the error analysis for the position of the detected features can be found in [9]. In order to take maximum benefit from the knowledge of the PDF's, a ML-estimation is performed. The PDF for the error of the position of a feature point is

$$
p(\mathbf{x} \mid \overline{\mathbf{x}})=\frac{e^{\left[-\frac{1}{2}(\mathbf{x}-\overline{\mathbf{x}})^{\top} \mathbf{C}^{-1}(\mathbf{x}-\overline{\mathbf{x}})\right]}}{2 \pi \sqrt{\operatorname{det}(\mathbf{C})}}
$$

where $\mathbf{x}$ is the measured point, $\overline{\mathbf{x}}$ is the true point and $\mathrm{C}$ the covariance matrix. Let

$$
\mathbf{z}=\left(\mathbf{x}^{(1)^{\top}}, \ldots, \mathbf{x}^{(M)^{\top}}\right)^{\top}
$$

be the vector of all points belonging to the estimated line. The task is to estimate the corresponding points $\hat{\mathbf{x}}^{(i)}$ on the line, so that the likelihood $L$

$$
L=\prod_{i} p\left(\mathbf{x}^{(i)} \mid \hat{\mathbf{x}}^{(i)}\right)
$$

is maximized.

For a specified line $(\phi, \rho)$, the estimated point $\hat{\mathbf{x}}^{(i)}$ can be determined directly. From the condition

$$
L \rightarrow \max
$$

follows

$$
p\left(\mathbf{x}^{(i)} \mid \hat{\mathbf{x}}^{(i)}\right) \rightarrow \max .
$$

This yields

$$
\frac{e^{\left[-\frac{1}{2}\left(\mathbf{x}^{(i)}-\hat{\mathbf{x}}^{(i)}\right)^{\top} \mathbf{C}^{(i)-1}\left(\mathbf{x}^{(i)}-\hat{\mathbf{x}}^{(i)}\right)\right]}}{2 \pi \sqrt{\operatorname{det}\left(\mathbf{C}^{(i)}\right)}} \rightarrow \max
$$

with the constraint that the point $\hat{\mathbf{x}}$ must lie on the line $(\phi, \rho)$. This constraint is expressed by

$$
\hat{\mathbf{x}}^{(i)}=\left(\begin{array}{c}
\rho \cos (\phi) \\
\rho \sin (\phi)
\end{array}\right)+\lambda^{(i)}\left(\begin{array}{c}
\sin (\phi) \\
-\cos (\phi)
\end{array}\right)=\mathbf{a}+\lambda^{(i)} \mathbf{b},
$$

where $\lambda^{(i)}$ is a scalar, $\mathbf{a}$ is a pointing vector and $\mathbf{b}$ is the direction. With this constraint and some additional simplifications, the condition (11) becomes

$$
\left(\mathbf{x}^{(i)}-\mathbf{a}-\lambda^{(i)} \mathbf{b}\right)^{\top} \mathbf{C}^{(i)^{-1}}\left(\mathbf{x}^{(i)}-\mathbf{a}-\lambda^{(i)} \mathbf{b}\right) \rightarrow \min .
$$

This minimization is satisfied by

$$
\frac{\partial}{\partial \lambda^{(i)}}\left(\mathbf{x}^{(i)}-\mathbf{a}-\lambda^{(i)} \mathbf{b}\right)^{\top} \mathbf{C}^{(i)}{ }^{-1}\left(\mathbf{x}^{(i)}-\mathbf{a}-\lambda^{(i)} \mathbf{b}\right)=0
$$

which yields

$$
\lambda^{(i)}=\frac{\left(\mathbf{x}^{(i)}-\mathbf{a}\right)^{\top} \mathbf{C}^{(i)}{ }^{-1} \mathbf{b}}{\mathbf{b}^{\top} \mathbf{C}^{(i)^{-1}} \mathbf{b}} .
$$

With

$$
\lambda^{(i)}=\lambda^{(i)}\left(\phi, \rho, \mathbf{x}^{(i)}\right)
$$

and

$$
\hat{\mathbf{x}}^{(i)}=\hat{\mathbf{x}}^{(i)}\left(\lambda^{(i)}\left(\phi, \rho, \hat{\mathbf{x}}^{(i)}\right)\right)
$$

the Likelihood results in the following cost function for the estimation of the line parameters $\phi, \rho$

$$
\sum_{i}^{M}\left[\mathbf{x}^{(i)}-\hat{\mathbf{x}}^{(i)}\left(\lambda^{(i)}\right)\right]^{\top} \mathbf{C}^{(i)}{ }^{-1}\left[\mathbf{x}^{(i)}-\hat{\mathbf{x}}^{(i)}\left(\lambda^{(i)}\right)\right] \rightarrow \min .
$$

The optimization can be done by iterative minimization methods.

\section{Propagation of error covari- ance}

In order to determine the impact of the collinearity on the accuracy of the camera parameters, the error covariances are propagated from the feature points up to the camera parameters. The propagation is started from the detected points up to the line in subsection 3.1 and continued from the line up to the corrected/projected points in subsection 3.2. 


\subsection{Error covariance of line parame- fied ters}

The cost function (18) has the form

$$
f(\hat{\phi}(\mathbf{z}), \hat{\rho}(\mathbf{z}) ; \mathbf{z})=\sum_{i=1}^{M}\left(\mathbf{d}^{(i)}\right)^{\top} \mathbf{C}^{(i)} \mathbf{d}^{(i)} \rightarrow \min
$$

with

$$
\mathbf{d}^{(i)}=\mathbf{x}^{(i)}-\hat{\mathbf{x}}^{(i)} \text {. }
$$

A necessary condition is that the gradient becomes zero

$$
\mathbf{h}=\operatorname{grad} f=\left.\frac{d}{d(\phi, \rho)} f(\phi(\mathbf{z}), \rho(\mathbf{z}) ; \mathbf{z})\right|_{\phi=\hat{\phi}, \rho=\hat{\rho}} \stackrel{!}{=} \mathbf{0} .
$$

It is not possible to resolve this equation for $(\hat{\phi}, \hat{\rho})$ algebraically in a trivial way. This means there is no closed form for a mapping $\mathbf{g}$ with

$$
\mathbb{R}^{2 M} \rightarrow \mathbb{R}^{2}: \underbrace{(\hat{\phi}, \hat{\rho})^{\top}}_{\in \mathbb{R}^{2}}=\mathbf{g}(\underbrace{\mathbf{z}}_{\in \mathbb{R}^{2 M}})
$$

available. On the other hand, the implicitly defined function $\mathbf{h}(\mathbf{g}(\mathbf{z}), \mathbf{z}) \stackrel{!}{=} \mathbf{0}$ enables the calculation of the Jacobian $\frac{d \mathbf{g}}{d \mathbf{z}}$ by utilizing the theorem about implicit functions in order to determine the first order approximation of the desired function

$$
\begin{aligned}
& \left(\begin{array}{ll}
\partial_{x_{1}(i)} g_{1} & \partial_{x_{2}(i)} g_{1} \\
\partial_{x_{1}(i)} g_{2} & \partial_{x_{2}(i)} g_{2}
\end{array}\right) \\
= & -\left(\begin{array}{ll}
\partial_{\phi} h_{1} & \partial_{\rho} h_{1} \\
\partial_{\phi} h_{2} & \partial_{\rho} h_{2}
\end{array}\right)^{-1}\left(\begin{array}{ll}
\partial_{x_{1}(i)} h_{1} & \partial_{x_{2}(i)} h_{1} \\
\partial_{x_{1}(i)} h_{2} & \partial_{x_{2}(i)} h_{2}
\end{array}\right)
\end{aligned}
$$

where $\partial_{a} \equiv \frac{\partial}{\partial_{a}}$ and $\partial_{a, b} \equiv \frac{\partial^{2}}{\partial_{a} \partial_{b}}$. This yields

$$
\begin{aligned}
\partial_{\mathbf{z}} \mathbf{g} & =-\left(\partial_{\mathbf{g}} \mathbf{h}\right)^{-1} \partial_{\mathbf{z}} \mathbf{h} \\
& =-\left(\partial_{\phi, \rho} \mathbf{h}\right)^{-1} \partial_{\mathbf{z}} \mathbf{h} .
\end{aligned}
$$

The linearized function is

$$
\begin{gathered}
\mathbf{g}(\mathbf{z}+\mathbf{e}) \approx \\
\left(\begin{array}{c}
\hat{\phi} \\
\hat{\rho}
\end{array}\right)+\underbrace{\left(\begin{array}{llll}
\partial_{x_{1}^{(1)}} g_{1} & \partial_{x_{2}^{(1)}} g_{1} \cdots \partial_{x_{1}^{(M)}} g_{1} \partial_{x_{2}^{(M)}} g_{1} \\
\partial_{x_{1}^{(1)}} g_{2} & \partial_{x_{2}^{(1)}} g_{2} \cdots \partial_{x_{1}^{(M)}} g_{2} & \partial_{x_{2}^{(M)}} g_{2}
\end{array}\right)}_{\mathrm{A}} \mathbf{e} \\
\mathbf{g}(\mathbf{z}+\mathbf{e}) \approx(\hat{\phi}, \hat{\rho})^{\top}+\mathrm{Ae} .
\end{gathered}
$$

After determining the first order approximation the error covariance of the line parameters can be speci-

$$
\operatorname{cov}(\hat{\phi}, \hat{\rho})=\Lambda=\mathrm{A}\left(\begin{array}{lllll}
\mathrm{C}_{11}^{(1)} & \mathrm{C}_{12}^{(1)} & & & \\
\mathrm{C}_{21}^{(1)} & \mathrm{C}_{22}^{(1)} & & & \\
& & \ddots & & \\
& & & \mathrm{C}_{11}^{(M)} & \mathrm{C}_{12}^{(M)} \\
& & & \mathrm{C}_{21}^{(M)} & \mathrm{C}_{22}^{(M)}
\end{array}\right) \mathrm{A}^{\top} .
$$

\subsection{Error covariance of projected point position}

In order to calculate the error covariance of the estimated points on the line the function $\mathbf{P}$, which is determined by equations (12) and (15)

$$
\hat{\mathbf{x}}=\mathbf{P}\left(\phi, \rho, \mathbf{x}^{(i)}\right)=\mathbf{a}(\hat{\phi}, \hat{\rho})+\lambda^{(i)}\left(\hat{\phi}, \hat{\rho}, \mathbf{x}^{(i)}\right) \mathbf{b}(\hat{\phi}, \hat{\rho}),
$$

is linearized by its first order taylor series

$$
\begin{aligned}
& \mathbf{P}\left(\left(\hat{\phi}, \hat{\rho}, x_{1}^{(i)}, x_{2}^{(i)}\right)^{\top}+\boldsymbol{\delta}\right) \approx \\
& \left(\begin{array}{c}
\hat{x}_{1}^{(i)} \\
\hat{x}_{2}^{(i)}
\end{array}\right)+\underbrace{\left(\begin{array}{c}
\partial_{\phi} \mathbf{P}_{1} \partial_{\rho} \mathbf{P}_{1} \partial_{x_{1}(i)} \mathbf{P}_{1} \partial_{x_{2}(i)} \mathbf{P}_{1} \\
\partial_{\phi} \mathbf{P}_{2} \partial_{\rho} \mathbf{P}_{2} \partial_{x_{1}(i)} \mathbf{P}_{2} \partial_{x_{2}(i)} \mathbf{P}_{2}
\end{array}\right)}_{\mathrm{B}^{(i)}\left(\phi, \rho, x_{1}{ }^{(i)}, x_{2}{ }^{(i)}\right)} \boldsymbol{\delta}
\end{aligned}
$$

where

$$
\boldsymbol{\delta}=\left(\begin{array}{c}
\delta \phi \\
\delta \rho \\
\delta x_{1}{ }^{(i)} \\
\delta x_{2}{ }^{(i)}
\end{array}\right)
$$

The error covariance of a projected point can be approximated by

$$
\operatorname{cov}\left(\hat{\mathbf{x}}^{(i)}\right)=\mathrm{B}^{(i)}\left(\begin{array}{cc}
\Lambda & 0 \\
0 & \mathrm{C}^{(i)}
\end{array}\right) \mathrm{B}^{(i)^{\top}}
$$

where

$$
\mathrm{B}^{(i)}=\mathrm{B}^{(i)}\left(\hat{\phi}, \hat{\rho}, x_{1}{ }^{(i)}, x_{2}{ }^{(i)}\right) .
$$

Figure 1 shows an example for the error ellipses before and after the projection of points.

As a result, which can be verified intuitively, the error covariance component perpendicular to the line shows maximal decrease whereas the component parallel to the line does not encounter any change. Furthermore, the outer points on the line remain higher error covariances than the more central points.

\section{Cramer-Rao bounds}

There are universal bounds for the accuracy of the estimated parameters determined by the CramerRao bounds. No estimator can yield parameter estimates which have lower error covariances than the 

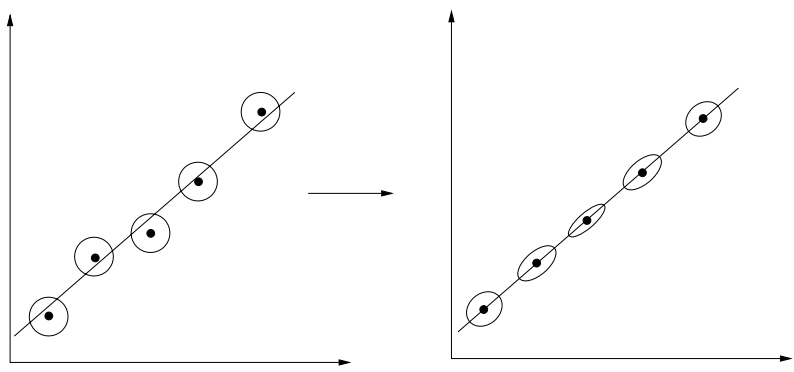

Figure 1: Point error ellipses before and after projection

Cramer-Rao bounds. In section 4.1 the error covariance bounds for the line parameters are determined and in section 4.2 the error covariance bounds for the projected point positions are specified.

\subsection{Cramer-Rao bounds for the error covariances of line parameters}

In order to calculate the lower bounds of the line parameter error covariances we need to determine the Fisher information matrix $F$ which is defined as

$$
\mathrm{F}=-\mathrm{E}\left[\partial_{\phi, \rho} \partial_{\phi, \rho}^{\top} \ln [p(\mathbf{z} \mid \hat{\phi}, \hat{\rho})]\right]
$$

where

$$
\partial_{\phi, \rho} \partial_{\phi, \rho}^{\top}
$$

is the operator generating the Hessian matrix of the second derivatives. The components of the Fisher information matrix are defined as

$$
\mathrm{F}_{m n}=-\mathrm{E}\left[\partial_{m} \partial_{n} \ln [p(\mathbf{z} \mid \hat{\phi}, \hat{\rho})]\right]
$$

where

$$
\partial_{1} \equiv \partial_{\phi}, \partial_{2} \equiv \partial_{\rho} .
$$

Replacing $p(\mathbf{z} \mid \hat{\phi}, \hat{\rho})$ yields

$$
\begin{aligned}
\mathbf{F}_{m n}= & -\mathrm{E}\left[\partial_{m} \partial_{n} \ln \left[\prod_{i}^{M} \frac{e^{\left[-\frac{1}{2}\left(\mathbf{d}^{(i)}\right)^{\top} \mathbf{C}^{(i)} \mathbf{d}^{(i)}\right]}}{2 \pi \sqrt{\operatorname{det}\left(\mathbf{C}^{(i)}\right)}}\right]\right] \\
= & \sum_{i}^{M} \frac{1}{2} \mathrm{E}\left[\partial_{m} \partial_{n}\left(\mathbf{d}^{(i)}\right)^{\top} \mathbf{C}^{(i)} \mathbf{d}^{(i)}\right] \\
= & \sum_{i}^{M} \frac{1}{2} \int\left\{\frac{e^{\left[-\frac{1}{2}\left(\hat{\mathbf{x}}-\mathbf{x}^{(i)}\right)^{\top} \mathbf{C}^{(i)-1}\left(\hat{\mathbf{x}}-\mathbf{x}^{(i)}\right)\right]}}{2 \pi \sqrt{\operatorname{det}(\mathbf{C})}}\right. \\
& {\left.\left[\partial_{m} \partial_{n}\left(\mathbf{d}^{(i)}\right)^{\top} \mathbf{C}^{(i)}-1 \mathbf{d}^{(i)}\right] \mathbf{d} \hat{\mathbf{x}}\right\} }
\end{aligned}
$$

The Cramer-Rao bounds are obtained by

$$
\operatorname{cov}(\hat{\phi}, \hat{\rho})_{m n} \geq\left[\mathrm{F}^{-1}\right]_{m n}
$$

As an example, in the case of isotropic covariance matrices for the point position error of the form

$$
\mathrm{C}=\left(\begin{array}{cc}
\sigma^{2} & 0 \\
0 & \sigma^{2}
\end{array}\right)
$$

the components of the Fisher information matrix result in

$$
\begin{aligned}
\mathrm{F}_{11}= & -\sum_{i} \frac{2\left(x_{2}^{(i)}\right)^{2} \cos (\phi)^{2}+x_{1}^{(i)} \cos (\phi) \rho}{\sigma^{2}} \\
& -\sum_{i} \frac{4 x_{2}^{(i)} x_{1}^{(i)} \cos (\phi) \sin (\phi)}{\sigma^{2}} \\
& -\sum_{i} \frac{\left(x_{2}^{(i)}\right)^{2}+x_{2}^{(i)} \sin (\phi) \rho}{\sigma^{2}} \\
& -\sum_{i} \frac{-2\left(x_{1}^{(i)}\right)^{2} \cos (\phi)^{2}\left(x_{1}^{(i)}\right)^{2}}{\sigma^{2}} \\
\mathrm{~F}_{12}= & -\sum_{i} \frac{\sin (\phi) x_{1}^{(i)}-\cos (\phi) x_{2}^{(i)}}{\sigma^{2}} \\
\mathrm{~F}_{22}= & -\sum_{i} \frac{1}{\sigma^{2}}
\end{aligned}
$$

\subsection{Cramer-Rao bounds for the error covariances of projected point po- sitions}

The projection mapping (29) is of the form

$$
\mathbf{P}=\mathbf{P}\left(\phi, \rho, x_{1}, x_{2}\right) .
$$

Since the Cramer-Rao bounds for $\hat{\phi}, \hat{\rho}$ are already determined and the true PDF of $x_{1}, x_{2}$ is assumed to be known, equation (3.30) from [5] is used in order to obtain

$$
\mathrm{F}(\mathbf{P}(\boldsymbol{\theta}))^{-1}=\left[\partial_{\boldsymbol{\theta}} \mathbf{P}(\boldsymbol{\theta})\right] \mathrm{F}(\boldsymbol{\theta})^{-1}\left[\partial_{\boldsymbol{\theta}} \mathbf{P}(\boldsymbol{\theta})\right]^{\top}
$$

where $\boldsymbol{\theta}=\left(\hat{\phi}, \hat{\rho}, x_{1}, x_{2}\right)^{\top}$ and $\mathrm{F}^{-1}(\boldsymbol{\theta})$ is the inverse Fisher information matrix containing the CramerRao bounds for the parameters $\hat{\phi}, \hat{\rho}, x_{1}, x_{2}$. Since the term $\partial_{\boldsymbol{\theta}} \mathbf{P}(\boldsymbol{\theta})$ is already defined in (30) as B, it may be written

$$
\mathrm{F}(\mathbf{P}(\boldsymbol{\theta}))^{-1}=\mathrm{B}(\boldsymbol{\theta}) \mathrm{F}(\boldsymbol{\theta})^{-1} \mathrm{~B}(\boldsymbol{\theta})^{\top} .
$$




\section{Maximum-Likelihood timation and covariance propagation of camera pa- rameters}

ML-estimation of the camera parameters is performed by bundle adjustment [4] where the 3Dfeature points and the camera parameters are estimated simultaneously. In order to determine the error covariances, a brief review of the basic principles of the estimation process are given.

Let there be $V$ views and $N$ 3D-points. The MLestimation is then defined by

$$
\sum_{i}^{V} \sum_{j}^{N} d\left(\mathbf{x}^{(i j)}, \hat{\mathrm{P}}^{(i)} \hat{\mathbf{X}}^{(j)}\right)_{\mathrm{C}^{(i j)}}^{2} \rightarrow \min
$$

where $\mathbf{x}^{(i j)}$ is the $j$-th $2 \mathrm{D}$-point of the $i$-th view, $\hat{\mathrm{P}}^{(i)}$ is the projection function of the $i$-th camera, $\hat{\mathbf{X}}^{(j)}=\left(\hat{X}_{1}^{(j)}, \hat{X}_{2}^{(j)}, \hat{X}_{3}^{(j)}\right)^{\top}$ is the $j$-th 3D-point and $d(\ldots)_{\mathbb{C}^{(i j)}}$ is the Mahalanobis distance according to the covariance matrix $\mathrm{C}^{(i j)}$. Let

$$
\begin{aligned}
\mathbf{z} & =\left(\mathbf{x}^{(11)}, \ldots, \mathbf{x}^{(1 N)}, \mathbf{x}^{(V 1)}, \ldots, \mathbf{x}^{(V N)}\right)^{\top}(48) \\
\mathbf{Z} & =\left(\mathbf{X}^{(1)}, \ldots, \mathbf{X}^{(N)}\right)^{\top}
\end{aligned}
$$

be the vectors of the measured 2D-points and the $3 \mathrm{D}$-points respectively. The parameterization of the camera shall be noted by

$$
\mathrm{P}^{(i)}=\mathrm{P}^{(i)}\left(\mathbf{q}^{(i)}\right), \mathbf{q} \in \mathbb{R}^{r},
$$

where $\mathbf{q}$ is the vector containing $r$ camera parameters to be estimated. The vector representing all parameters can be noted as

$$
\mathbf{Q}=\left(q_{1}^{(1)}, \ldots, q_{r}^{(V)}, X_{1}^{(1)}, \ldots, X_{3}^{(N)}\right)^{\top} .
$$

This means for a parameter vector $\mathbf{Q}$ there are $2 \mathrm{D}$ points collected in $\hat{\mathbf{z}}$

$$
\begin{aligned}
f(\hat{\mathbf{Q}}) & =\left(\hat{\mathrm{P}}^{(1)}\left(\hat{\mathbf{q}}^{(1)}\right) \mathbf{X}^{(1)}, \ldots, \hat{\mathrm{P}}^{(N)}\left(\hat{\mathbf{q}}^{(N)}\right) \mathbf{X}^{(N)}\right)^{\top}(52) \\
& =\left(\hat{\mathbf{x}}^{(1)}, \ldots, \hat{\mathbf{x}}^{(N)}\right)^{\top} \\
& =\hat{\mathbf{z}}
\end{aligned}
$$

which are determined by $f(\hat{\mathbf{Q}})$. By collecting the covariance matrices as

$$
\Sigma=\left(\begin{array}{ccc}
\mathrm{C}^{(11)} & & \\
& \ddots & \\
& & \mathrm{C}^{(V N)}
\end{array}\right),
$$

the covariance of the estimated parameters is obtained [4] by

$$
\operatorname{cov}(\hat{\mathbf{Q}})=\left(\mathrm{J}^{\top} \Sigma^{-1} \mathrm{~J}\right)^{-1}
$$

$$
\mathrm{J}=\left.\frac{d f}{d \mathbf{Q}}\right|_{\mathbf{Q}=\hat{\mathbf{Q}}} .
$$

In order to determine the covariance in presence of collinear points, each collinear point $\mathbf{x}^{(i j)}$ is replaced by its estimated point $\hat{\mathbf{x}}^{(i j)}$ and each covariance matrix $\mathrm{C}^{(i j)}$ is replaced by the covariance matrix of $\hat{\mathbf{x}}^{(i j)}$ as determined in (32).

\section{Experimental results}

To demonstrate the impact of collinear features on the expected analytic covariances, camera parameter estimation is performed utilizing synthetic 3D-points which are regularly positioned within a cube, as shown in figure 2 . There are 12 line segments detected, vertical ones as well as horizontal ones. Therefore, the error of corrected points shows a consecutive decrease of covariance in both directions. In the following, results are shown for tests done with increasing std. deviations for the position error of the 2D-points, which are determined in 2 camera views. Each plot shows two curves for the parameter error: one with and one without exploiting the collinearity of the 2D-points.

Figures 3 and 4 show the results for the error variance of camera rotation and normalized translation, respectively. The normalized translation error variance is calculated from the translation unit vector. Figures 5 and 6 show the RMSE and the 3D-reconstruction error variance, respectively.

In all cases, exploiting the collinearity results in a considerable decrease in the error of camera parameters.

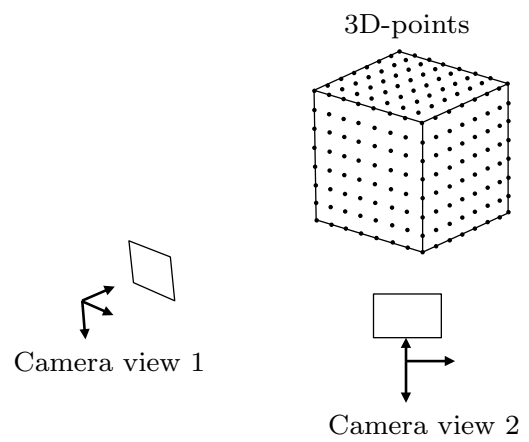

Figure 2: Synthetic camera and 3D-point setup 


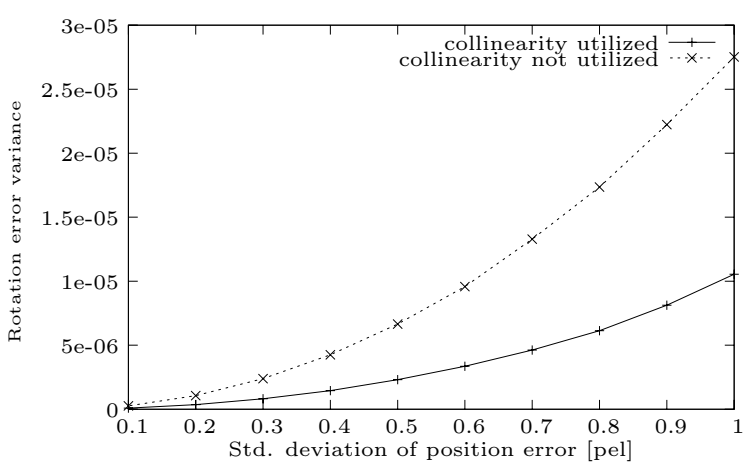

Figure 3: Rotation angle error variance

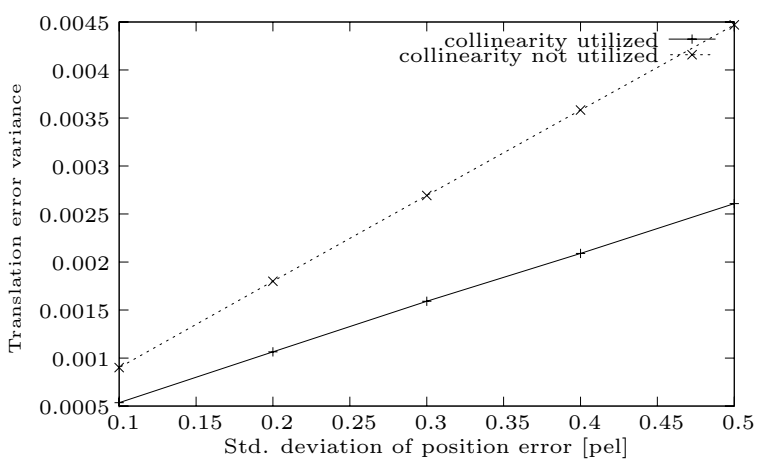

Figure 4: Normalized translation error variance

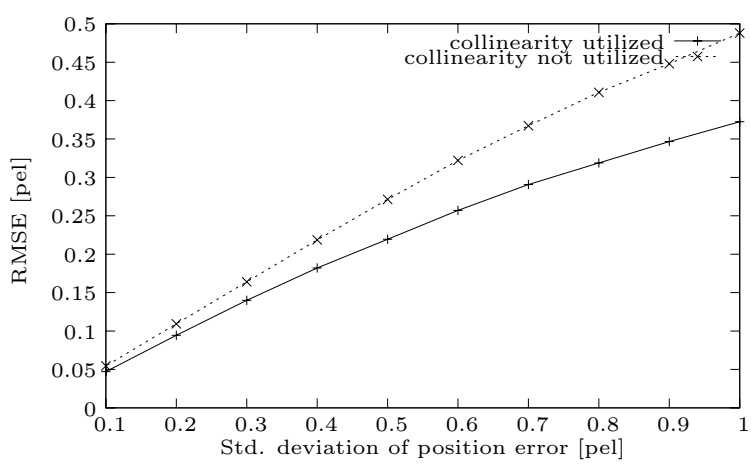

Figure 5: RMSE

\section{Conclusions}

An analytical error covariance propagation for camera parameter estimation in presence of collinear feature points is presented. The ML-estimation of the supporting lines and the corresponding covariance propagation is reviewed. The correction of collinear points with their covariances is theoretically determined, where interesting properties of error covariance decrease are shown.

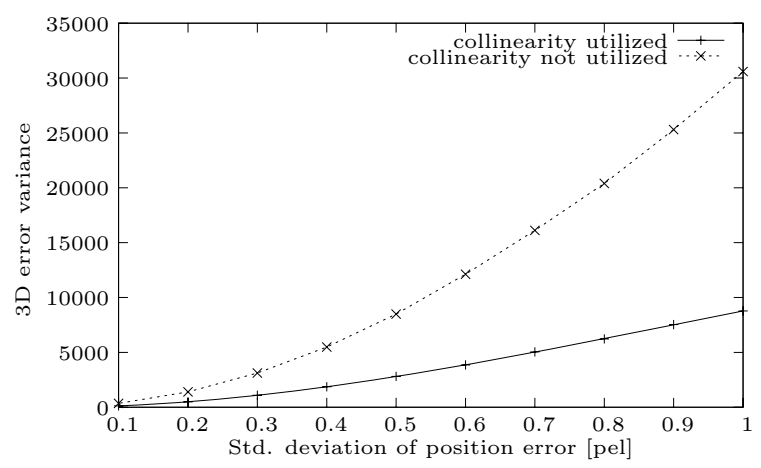

Figure 6: 3D-reconstruction error variance

By determining the Fisher information matrix the lower bounds for the error covariance of the corrected point positions are obtained.

This paper shows analytically as well as experimentally how much the accuracy of camera parameters can be increased by taking advantage of the information about the collinearity.

\section{Future work}

As shown in the experiments, some special constellations such as a mesh-like distribution of points, where one point shares more than one line might be modelled accordingly. In this case, a pencil-of-lines approach like in [8] would be appropriate where all lines which share points with other lines would have to be grouped and estimated simultaneously.

\section{References}

[1] A. Bartoli, M. Coquerelle, and P. Sturm. A framework for pencil-of-points structure-frommotion. In European Conference on Computer Vision, volume 2, pages 28-40. Springer, May 2004.

[2] R.O. Duda and P.E. Hart. Pattern Classification and Scene Analysis. John Wiley \& Sons, 1973.

[3] D. Forsyth and J. Ponce. Computer Vision: A Modern Approach. Prentice Hall, Upper Saddle River, New Jersey, 2000.

[4] Richard I. Hartley and Andrew Zisserman. Multiple View Geometry. Cambridge University Press, 2000. 
[5] S. M. Kay. Fundamentals of Statistical Signal Processing, Volume I, Estimation Theory. Prentice Hall, Upper Saddle River, 1993.

[6] G. Liu, Reinhard Klette, and Bodo Rosenhahn. Structure from motion in the presence of noise. In Image and Vision Computing New Zealand, 2005.

[7] Jianbo Shi and Carlo Tomasi. Good features to track. In IEEE Conference on Computer Vision and Pattern Recognition, pages 593-600, 1994.

[8] G. Speyer and M. Werman. Parameter estimates for a pencil of lines: Bounds and estimators. In European Conference on Computer Vision, pages 432-446. Springer, 2002. Copenhagen.

[9] Richard Szeliski. Bayesian Modeling of Uncertainty in Low-Level Vision. Kluwer Academic Publishers, Boston, 1989.

[10] Carlo Tomasi and Takeo Kanade. Detection and tracking of point features, April 1991. Carnegie Mellon University Technical Report CMU-CS91-132. 